\title{
LEVELS OF CARBOXYLATED \\ AND UNDERCARBOXYLATED OSTEOCALCIN IN PATIENTS WITH TYPE 2 DIABETES
}

\author{
Silvia Gancheva ${ }^{1}$, Elena Marinova ${ }^{2}$, Branimir Kanazirev ${ }^{2}$, Mila Boyadzhieva ${ }^{3}$, \\ Maria Zhelyazkova-Savova ${ }^{1}$ \\ ${ }^{1}$ Department of Pharmacology and Clinical Pharmacology and Therapeutics, \\ Faculty of Medicine, Medical University of Varna \\ ${ }^{2}$ Department of Propaedeutics of Internal Medicine, Faculty of Medicine, \\ Medical University of Varna \\ ${ }^{3}$ Second Department of Internal Diseases, Faculty of Medicine, \\ Medical University of Varna
}

\begin{abstract}
INTRODUCTION: Osteocalcin (OC) is a bone-derived protein that undergoes vitamin K-dependent carboxylation. The undercarboxylated form of the protein (ucOC) is released in the circulation during the process of bone resorption. Experimental studies on mice and rats have revealed that $\mathrm{ucCC}$ is involved in the regulation of energy homeostasis, linking in this way the bone, pancreas, and adipose tissue metabolism. Experimental studies suggest no hormonal role for the carboxylated form (cOC) of the protein.

AIM: In the current study we aimed to examine the levels of $\mathrm{OC}$ in its carboxylated and undercarboxylated form in patients with type 2 diabetes and control subjects, and to compare the vitamin $K$ status between the two groups.

MATERIALS AND METHODS: The present cross-sectional study involved a sample of 46 adults type 2 diabetes patients and a control group of 19 individuals. The carboxylated and undercarboxylated forms of OC were measured in serum by using highly sensitive sandwich-type enzyme immunoassay kits. Vitamin $\mathrm{K}$ status was evaluated by the ratio ucOC/cOC. Student's two-tailed unpaired t-test was used to compare the groups.

RESULTS: UcOC and cOC serum levels were significantly lower in patients with type 2 diabetes compared to controls. We found no difference in the vitamin $\mathrm{K}$ status between the groups.

CONCLUSION: Our results show that $\mathrm{OC}$ might be involved in the regulation of carbohydrate metabolism. In humans, it appears that the carboxylation state might not be essential for the hormonal role of the protein as in mice and rats.
\end{abstract}

Keywords: carboxylated osteocalcin, undercarboxylated osteocalcin, type 2 diabetes, vitamin $K$ status

Address for correspondence:

Silvia Gancheva

Faculty of Medicine

Medical University of Varna

55 Marin Drinov St

9002 Varna

e-mail: silvia.gancheva@mu-varna.bg

Received: November 23, 2020

Accepted: December 8, 2020

\section{INTRODUCTION}

Osteocalcin (OC) is the most abundant noncollagenous protein in the bone matrix. It is synthesized by osteoblasts. Its molecule contains three gamma-glutamic acid residues undergoing posttranslational vitamin K-dependent carboxylation. The carboxylated residues bind to calcium ions in the hydroxyapatite crystals with high affinity and incor- 
Silvia Gancheva, Elena Marinova, Branimir Kanazirev et al.

porate the protein in the bone matrix (1). It has been long thought that the only physiological role of OC is to regulate bone mineralization and that, like the other vitamin $\mathrm{K}$ dependent proteins, $\mathrm{OC}$ is active in its carboxylated form (cOC). The traditional concept postulates that the circulating levels of OC reflects the bone turnover rate. It is laboratory measured to predict the fracture risk and assess the effectiveness of antiresorptive therapy $(2,3)$.

In a state of vitamin $\mathrm{K}$ deficiency, the reaction of carboxylation is impaired, resulting in a rise in the levels of ucOC. In contrast, vitamin K supplementation reduces the fraction of ucOC (4). Therefore, the ratio between the undercarboxylated and carboxylated form of the protein is assumed to be an inverse marker of vitamin K status (5).

In 1996, an American research group, aiming to clarify the role of OC in bone, generated OC knockout mice (6). The result was surprising - the OC deficient animals were obese, had impaired glucose metabolism and higher bone mass (7). Since then, multiple studies have confirmed and further developed the concept that OC regulates energy homeostasis and thus links the bone, pancreas, and adipose tissue in a regulatory loop. OC has been qualified as a hormone, secreted by bone and acting on pancreatic beta cells and adipocytes. OC is decarboxylated during the process of bone resorption and released into the systemic circulation in its undercarboxylated form (ucOC). It was suggested that ucOC was mediating the metabolic function of this hormone (7). This was later confirmed by showing that intermittent injections of ucOC was able to improve glucose metabolism and to prevent type 2 diabetes in mice (8).

In a previous study of ours, we tested whether OC would behave in a similar manner also in rats. We measured the levels of both OC forms in Wistar rats with high-fat high-fructose (HFHF) diet-induced metabolic syndrome and found serum concentration of ucOC to be lower in the metabolic animals than in the controls. There was no difference in the cOC concentration (9). We next showed that the pharmacological reduction of ucOC level by chronic alendronate treatment was associated with impairment of glucose metabolism in intact rats and rats fed HFHF diet (10). Thus, we provided evidence that the hormonal function of ucOC could be extended to a different rodent species, while cOC seemed to be inactive.

The experimental findings of a hormonal role for OC were challenging for numerous clinical studies that aimed to extrapolate it to humans. The hypothesis that OC is involved in the pathogenesis of obesity, metabolic syndrome, and diabetes type 2 was tested repeatedly, but the results were less consistent. Some studies confirmed the hormonal activity of ucOC (11-13), others demonstrated beneficial metabolic roles for total OC and/or cOC as well $(14,15)$.

The notion that only ucOC is endowed with metabolic activity implies that vitamin $\mathrm{K}$ would be associated with detrimental effects on carbohydrate metabolism, as it is involved in the carboxylation process (16). However, epidemiological and clinical data suggest that vitamin $\mathrm{K}$ is an important marker of good bone and cardio-metabolic health (17-22). Moreover, it is considered that many of the diseases of aging with social impact can be regarded as a form of subclinical vitamin $\mathrm{K}$ deficiency, which has been found to be widely distributed among the Western population (18).

\section{AIM}

The aim of the current study was to examine the levels of OC in its carboxylated and undercarboxylated form in patients with type 2 diabetes and control subjects and to compare the vitamin $\mathrm{K}$ status between the two groups.

\section{MATERIALS AND METHODS}

\section{Participants}

This cross-sectional clinical study involved a sample of 46 adults (25 females and 21 males) with type 2 diabetes without cardiovascular complications that were hospitalized in the St. Marina University Hospital, Varna in the period October 2018 - November 2019, and a control group of 19 subjects (11 females and 8 males). The study was approved by the local Ethical Committee of the Medical University of Varna.

\section{Measurement of Osteocalcin Concentration}

Serum of the patients and control participants was collected and stored at $-60^{\circ} \mathrm{C}$ for biochemical determination of OC concentration. Highly sensitive sandwich-type enzyme immunoassay (EIA) kits for ucOC and cOC were used (Takara Bio, Inc., Japan), 
following the producer's instructions. Every kit utilized two mouse monoclonal antibodies against OC, one of which was coated onto the plate and the other was peroxidase-labeled. The first one recognized specifically the measured protein (ucOC or $\mathrm{COC}$, respectively). The peroxidase-labeled anti-OC monoclonal antibody was required for the reaction between peroxidase and the substrate added $\left(\mathrm{H}_{2} \mathrm{O}_{2}\right.$ and tetramethylbenzidine) resulting in color development with intensities proportional to the respective amount of ucOC and COC present in the samples. The amount of the proteins was quantitated by measuring the absorbance using an ELISA reader LKB 5060-006 (LKB Instruments, Australia) at $450 \mathrm{~nm}$.

A

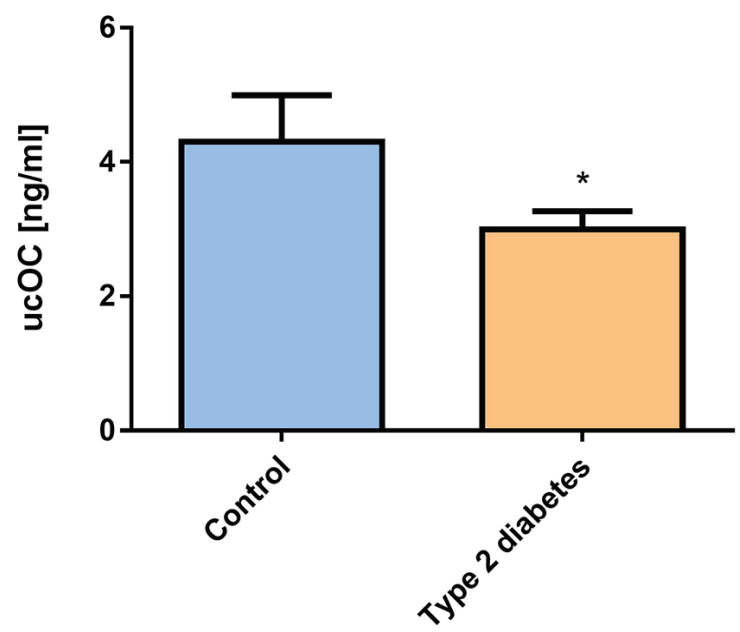

\section{Statistics}

Results were presented as a mean \pm standard error of the mean (SEM). The groups were compared by Student's two-tailed unpaired t-test. Differences were considered significant at $\mathrm{p}<0.05$. The statistical software GraphPad Prism 5 was used (GraphPad Software, Inc.).

\section{RESULTS}

The levels of ucOC and cOC are presented on Fig. 1. The concentration of ucOC (Fig. 1A) in the serum of controls was $4.301 \pm 0.69 \mathrm{ng} / \mathrm{mL}$. The patients with type 2 diabetes had significantly lower level of ucOC $-2.994 \pm 0.27 \mathrm{ng} / \mathrm{mL}(\mathrm{p}=0.0365)$. The serum level of cOC (Fig. 1B) was $9.839 \pm 0.10 \mathrm{ng} / \mathrm{mL}$ in the

B

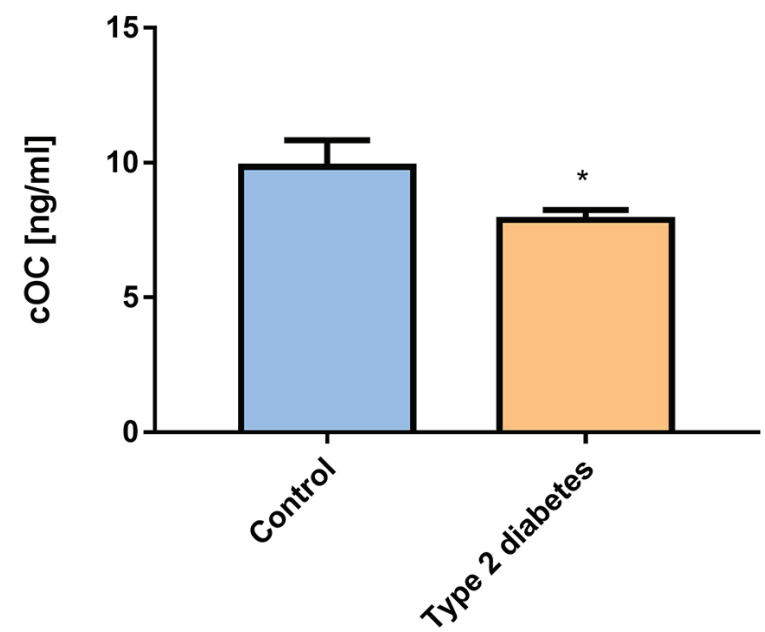

Fig. 1. Levels of $u c O C$ and $c O C$

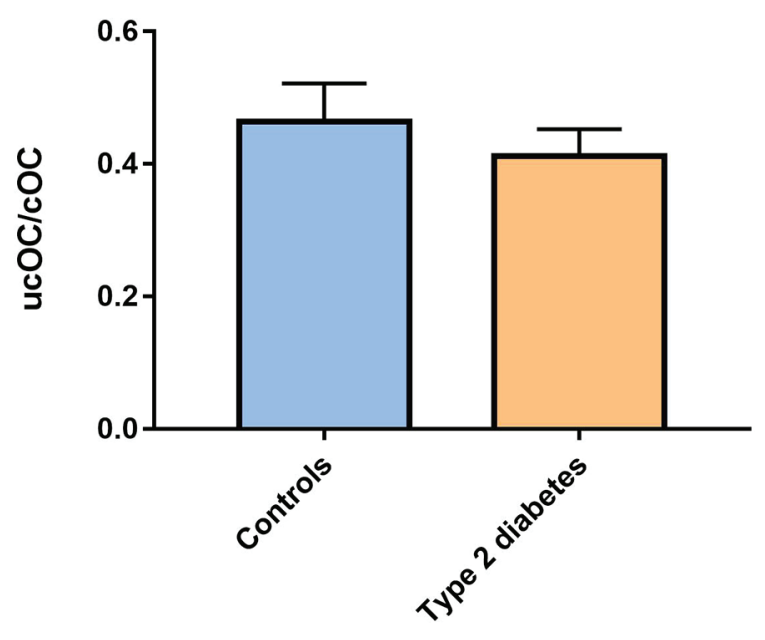

Fig. 2. Ratio of $u c O C$ and $c O C$ control group and significantly lower in the diabetic group $-7.883 \pm 0.36 \mathrm{ng} / \mathrm{mL}$ ( $\mathrm{p}=0.0237)$.

The ratio of ucOC and cOC, used as an indicator of vitamin $\mathrm{K}$ status, is presented on Fig. 2. It was $0.4643 \pm 0.06$ in the control group and $0.4117 \pm 0.04$ in the diabetic group, the difference being insignificant $(\mathrm{p}=0.4835)$.

\section{DISCUSSION}

The current study demonstrated that both ucOC and cOC levels were reduced in our diabetic patients. Our results partly agree with those of Sanchez-Enriquez et al. (11) and Takaya et al. (23) who report, similarly to us, lower levels of ucOC in pa- 
Silvia Gancheva, Elena Marinova, Branimir Kanazirev et al.

tients with type 2 diabetes compared to controls. A recent research, involving patients with metabolic syndrome without type 2 diabetes, found that the patients with ucOC levels below the $25^{\text {th }}$ percentile showed worse cardiometabolic profile and higher cardiovascular and type 2 diabetes risk. Therefore, the authors suggest that the circulating ucOC could serve as a tool to estimate the cardiovascular and type 2 diabetes risk in patients with metabolic syndrome (12). In contrast, Thrailkill et al. compared the levels of both forms of OC in patients with type 1 diabetes and healthy controls and found no differences between the groups. However, the authors established an association of ucOC serum concentrations with either endogenous production or exogenous administration of insulin. The authors assume that ucOC is, at least in part, regulated through insulin-mediated events (24). Such a regulatory mechanism was demonstrated in mouse and human osteoblasts by Ferron et al. (25). Insulin, by activating its receptors on osteoblast membrane, stimulated bone resorption thus promoting OC decarboxylation and release into the systemic circulation. The decreased insulin sensitivity in type 2 diabetes impaired insulin-dependent regulation of OC release and resulted in reduced circulating level of ucOC. In patients with type $1 \mathrm{di}-$ abetes, insulin sensitivity was generally unaltered. The preserved function of insulin receptors may explain the lack of differences in ucOC levels between diabetic patients and controls observed by Thrailkill et al. (24). According to the experiments conducted on OC deficient mice, ucOC, by activating specific G-protein coupled receptors in pancreatic beta cells, stimulates insulin secretion and beta-cell proliferation (26). Such receptors have not been proven thus far in human beta-cells. However, many clinical studies, summarized in a meta-analysis by Liu et al., have revealed a negative correlation of serum ucOC concentration with fasting blood glucose and glycated hemoglobin level (13). The action of ucOC on human beta cells was confirmed by Sabek et al. (27) and Thrailkill et al. (24) who found association of higher ucOC levels with markers of endogenous insulin secretion (C-peptide and C-peptide/glucose ratio). In summary, the majority of clinical trials support the statement that ucOC acts as a bone-derived regulator of glucose homeostasis, and can be used as a marker of impaired carbohydrate metabolism.
Because experimental research suggests that ucOC is the hormonally active form of OC (7), most of the latest clinical studies are focused on ucOC serum concentration and its impact on the glucose homeostasis in humans. However, earlier studies have utilized more frequently the total OC; the abovementioned meta-analysis (13) concludes that total OC is also negatively correlated with fasting plasma glucose and HbAlc in humans.

Less data is available about the potential connection of cOC with glucose homeostasis. Shea et al. report an association of elevated $\mathrm{COC}$, but not ucOC levels with lower insulin resistance in nondiabetic patients (15). An association of cOC with improved insulin sensitivity is reported also by Hwang et al., who found that both $\mathrm{COC}$ and ucOC were associated with improved glucose tolerance. According to this study, the ucOC level is associated with enhanced beta-cell function, and $\mathrm{COC}$ - with improved insulin sensitivity in middle-aged male subjects (14). In agreement with these data, Razny et al. found that the cOC level was reduced in obese compared to nonobese subjects, and that ucOC was lower in prediabetic individuals compared to healthy obese volunteers (28). Knapen et al. (29) demonstrated that higher carboxylation of OC was significantly correlated with lower body weight, BMI, and fat mass of the trunk in healthy postmenopausal women. Lu et al. (30) conducted a family-based study across three generations on non-diabetic women to examine the age-specific associations of OC forms with glucose and adipokines. Among the few correlations found was the inverse one between $\mathrm{COC}$ and leptin in mothers.

In the current study, we have established that both forms of OC were lower in patients with type 2 diabetes compared to controls. These results suggest that in humans the action of OC might be independent of its carboxylation status.

Vitamin $\mathrm{K}$ dietary intake affects the process of OC carboxylation. Truong et al. have compared the ucOC levels, presented as a percentage of the total OC, in volunteers at different age consuming a phylloquinone-restricted diet followed by a phylloquinone-supplemented diet. The authors report that in all participants in the study, regardless of age and sex, ucOC percentage was increased by phylloquinone depletion and decreased by phylloquinone re- 
pletion (4). Observational studies report that high dietary vitamin $\mathrm{K}$ intake and circulating phylloquinone level are associated with reduced risk of obesity (19) and type 2 diabetes (20-22). Vitamin K supplementation improves the carbohydrate metabolism in healthy subjects of different age and in patients with prediabetes or diabetes type $2(31,32)$, but evidence shows that vitamin $\mathrm{K}$ can have beneficial effects also through mechanisms different from gamma-glutamic carboxylation (33). In our study, we looked for a difference in the vitamin $\mathrm{K}$ status between the control and the diabetic group by comparing the ucOC/ cOC ratio. We hypothesized that the patients with diabetes would present with subclinical vitamin $\mathrm{K}$ deficiency and, correspondingly, we expected a higher ucOC/cOC ratio in the diabetic group vs. the control subjects. However, no such difference in the vitamin K status was found between the studied groups.

\section{CONCLUSION}

In the current study, the levels of both carboxylated and undercarboxylated osteocalcin were reduced in patients with type 2 diabetes compared to controls. Our results confirmed the involvement of this bone-derived protein in the regulation of carbohydrate metabolism. In humans, it is possible that the state of carboxylation may not be the determinant factor for the hormonal role of osteocalcin.

\section{Acknowledgements}

The study is supported by the Science Fund of Medical University of Varna (Grant № 17001).

\section{REFERENCES}

1. Hauschka PV, Lian JB, Cole DE, Gundberg CM. Osteocalcin and matrix Gla protein: vitamin Kdependent proteins in bone. Physiol Rev. 1989; 69(3):990-1047.

2. Atalay S, Elci A, Kayadibi H, Onder CB, Aka N. Diagnostic utility of osteocalcin, undercarboxylated osteocalcin, and alkaline phosphatase for osteoporosis in premenopausal and postmenopausal women. Ann Lab Med. 2012;32(1):23-30. doi: 10.3343/ alm.2012.32.1.23.

3. Jagtap VR, Ganu JV, Nagane NS. BMD and serum intact osteocalcin in postmenopausal osteoporosis women. Indian J Clin Biochem. 2011; 26(1):70-3. doi: 10.1007/s12291-010-0074-2.

4. Truong JT, Fu X, Saltzman E, Al Rajabi A, Dallal GE, Gundberg CM, et al. Age group and sex do not influence responses of vitamin $\mathrm{K}$ biomarkers to changes in dietary vitamin K. J Nutr. 2012;142(5):936-41. doi: 10.3945/jn.111.154807.

5. Shea MK, Booth SL. Concepts and controversies in evaluating vitamin $\mathrm{K}$ status in populationbased studies. Nutrients. 2016; 8(1):8. doi:10.3390/ nu8010008.

6. Ducy P, Desbois C, Boyce B, Pinero G, Story B, Dunstan C, et al. Increased bone formation in osteocalcin-deficient mice. Nature. 1996; 382(6590):448-52. doi: 10.1038/382448a0.

7. Lee N, Sowa H, Hinoi E, Ferron M, Ahn J, Confavreux $\mathrm{C}$, et al. Endocrine regulation of energy metabolism by the skeleton. Cell. 2007; 130(3):456-69. doi: 10.1016/j.cell.2007.05.047.

8. Ferron M, McKee M, Levine R, Ducy P, Karsenty G. Intermittent injections of osteocalcin improve glucose metabolism and prevent type 2 diabetes in mice. Bone. 2012;50(2):568-75. doi: 10.1016/j. bone.2011.04.017.

9. Gancheva S, Galunska B, Zhelyazkova-Savova M. Osteocalcin in a rat model of metabolic syndrome. Adipobiology. 2015; 7:31-6. doi: 10.14748/adipo. v7.1558.

10. Gancheva S, Zhelyazkova-Savova M. Are bisphosphonates associated with adverse metabolic and cognitive effects? A study in intact rats and rats fed high-fat high-fructose diet. Calcif Tissue Int. 2020; 107(1):41-51. doi: 10.1007/s00223-020-00684-5.

11. Sanchez-Enriquez S, Ballesteros-Gonzalez IT, Villafán-Bernal JR, Pascoe-Gonzalez S, Rivera-Leon EA, Bastidas-Ramirez BE, et al. Serum levels of undercarboxylated osteocalcin are related to cardiovascular risk factors in patients with type 2 diabetes mellitus and healthy subjects. World J Diabetes. 2017;8(1):11-7. doi: 10.4239/wjd.v8.i1.11.

12. Riquelme-Gallego B, García-Molina L, CanoIbáñez N, Sánchez-Delgado G, Andújar-Vera F, García-Fontana C, et al. Circulating undercarboxylated osteocalcin as estimator of cardiovascular and type 2 diabetes risk in metabolic syndrome patients. Sci Rep. 2020;10(1):1840. doi: 10.1038/ s41598-020-58760-7.

13. Liu DM, Guo XZ, Tong HJ, Tao B, Sun LH, Zhao HY, et al. Association between osteocalcin and glucose metabolism: a meta-analysis. Osteoporos Int. 2015;26(12):2823-33. doi: 10.1007/ s00198-015-3197-8. 
Silvia Gancheva, Elena Marinova, Branimir Kanazirev et al.

14. Hwang Y, Jeong I, Ahn K, Chung H. The uncarboxylated form of osteocalcin is associated with improved glucose tolerance and enhanced betacell function in middle-aged male subjects. Diabetes Metab Res Rev. 2009;25(8):768-72. doi: 10.1002/ dmrr.1045.

15. Shea M, Gundberg C, Meigs J, Dallal G, Saltzman E, Yoshida M, et al. Gamma-carboxylation of osteocalcin and insulin resistance in older men and women. Am J Clin Nutr. 2009;90(5):1230-5. doi: 10.3945/ajcn.2009.28151.

16. Gundberg CM, Lian JB, Booth SL. Vitamin Kdependent carboxylation of osteocalcin: friend or foe? Adv Nutr. 2012;3(2):149-57. doi: 10.3945/ an.112.001834.

17. Zhelyazkova-Savova M, Gancheva S, Galunska B, Gerova D. Vitamin K: The new faces of an old friend - a role in bone and vascular health. Biomed Rev. 2017; 28:70-90. doi: 10.14748/bmr.v28.4453

18. McCann JC, Ames BN. Vitamin $\mathrm{K}$, an example of triage theory: is micronutrient inadequacy linked to diseases of aging? Am J Clin Nutr. 2009;90(4):889-907. doi: 10.3945/ajcn.2009.27930.

19. 19. Pan Y, Jackson R. Dietary phylloquinone intakes and metabolic syndrome in US young adults. J Am Coll Nutr. 2009;28(4):369-79. doi: 10.1080/07315724.2009.10718099.

20. Beulens J, van der A D, Grobbee D, Sluijs I, Spijkerman A, van der Schouw Y. Dietary phylloquinone and menaquinones intakes and risk of type 2 diabetes. Diabetes Care. 2010;33(8):1699-705. doi: 10.2337/dc09-2302.

21. Ibarrola-Jurado N, Salas-Salvado J, Martinez-Gonzalez M, Bullo M. Dietary phylloquinone intake and risk of type 2 diabetes in elderly subjects at high risk of cardiovascular disease. Am J Clin Nutr. 2012;96(5):1113-8. doi: 10.3945/ajcn.111.033498.

22. Zwakenberg SR, Remmelzwaal S, Beulens JWJ, Booth SL, Burgess S, Dashti HS, et al. Circulating phylloquinone concentrations and risk of type 2 diabetes: A Mendelian randomization study. Diabetes. 2019;68(1):220-5. doi: 10.2337/db18-0543.

23. Takaya J, Tanabe Y, Kuroyanagi Y, Kaneko K. Decreased undercarboxylated osteocalcin in children with type 2 diabetes mellitus. J Pediatr Endocrinol Metab. 2016;29(8):879-84. doi: 10.1515/ jpem-2015-0417.

24. Thrailkill KM, Jo CH, Cockrell GE, Moreau CS, Lumpkin CK Jr, Fowlkes JL. Determinants of undercarboxylated and carboxylated osteocalcin concentrations in type 1 diabetes. Osteoporos Int. 2012;23(6):1799-806. doi: 10.1007/ s00198-011-1807-7.

25. Ferron M, Wei J, Yoshizawa T, Del Fattore A, DePinho R, Teti A, et al. Insulin signaling in osteoblasts integrates bone remodeling and energy metabolism. Cell. 2010;142(2):296-308. doi: 10.1016/j. cell.2010.06.003.

26. Wei J, Hanna T, Suda N, Karsenty G, Ducy P. Osteocalcin promotes beta-cell proliferation during development and adulthood through Gprc6a. Diabetes. 2014;63(3):1021-31. doi: 10.2337/db13-0887.

27. Sabek OM, Redondo MJ, Nguyen DT, Beamish CA, Fraga DW, Hampe CS, et al. Serum C-peptide and osteocalcin levels in children with recently diagnosed diabetes. Endocrinol Diabetes Metab. 2019;3(1):e00104. doi: 10.1002/edm2.104.

28. Razny U, Fedak D, Kiec-Wilk B, Goralska J, Gruca A, Zdzienicka A, et al. Carboxylated and undercarboxylated osteocalcin in metabolic complications of human obesity and prediabetes. Diabetes Metab Res Rev. 2017;33(3):e2862. doi: 10.1002/dmrr.2862.

29. Knapen MHJ, Schurgers LJ, Shearer MJ, Newman $\mathrm{P}$, Theuwissen E, Vermeer C. Association of vitamin $\mathrm{K}$ status with adiponectin and body composition in healthy subjects: uncarboxylated osteocalcin is not associated with fat mass and body weight. Br J Nutr. 2012; 108(6):1017-24. doi: 10.1017/ S000711451100626X.

30. Lu C, Ivaska KK, Alen M, Wang Q, Törmäkangas T, Xu L, et al. Serum osteocalcin is not associated with glucose but is inversely associated with leptin across generations of nondiabetic women. J Clin Endocrinol Metab. 2012;97(11):4106-14. doi: 10.1210/jc.2012-2045.

31. Manna P, Kalita J. Beneficial role of vitamin K supplementation on insulin sensitivity, glucose metabolism, and the reduced risk of type 2 diabetes: A review. Nutrition. 2016; 32(7-8):732-9. doi: 10.1016/j. nut.2016.01.011.

32. Li Y, Chen JP, Duan L, Li S. Effect of vitamin K2 on type 2 diabetes mellitus: A review. Diabetes Res Clin Pract. 2018;136:39-51. doi: 10.1016/j. diabres.2017.11.020.

33. Ho H-J, Komai M, Shirakawa H. Beneficial effects of vitamin $\mathrm{K}$ status on glycemic regulation and diabetes mellitus: A mini-review. Nutrients. 2020; 12(8):2485. doi: 10.3390/nu12082485. 